\title{
Window-based Cooperative Caching Scheme for Streaming Multimedia Contents in Mobile Ad Hoc Networks
}

\author{
Backhyun Kim ${ }^{1}$ and Kyeongmo Park ${ }^{2+}$ \\ ${ }^{1}$ Faculty of Liberal Education, Incheon National University, \\ 119 Academy-ro, Yeonsu-gu, Incheon, Korea \\ ${ }^{2+}$ Department of Computer Science and Information Engineering, \\ The Catholic University of Korea, 43 Jibong-ro, Bucheon-si, Gyeonggi-do, Korea
}

\begin{abstract}
Mobile ad-hoc networks (MANETs) are networks comprised of nodes only. Due to the nature of network, energy and communication bandwidth are limited. In the case of MANETs, as nodes move freely, the links between nodes change from moment to moment and, in some cases, a black hole phenomenon where the service is disconnected occurs. Caching is a scheme of storing in advance and using the contents expected to be required later. The caching scheme can improve service delay and service disconnection caused by a black hole phenomenon, etc. The performance of caching depends on which nodes perform caching and which nodes are caching the requested content. In this paper, the contents being transmitted are cached in the node that comprises the transmission path and the requesting node. For streaming service without delay, the client node requests and caches the contiguous frames required for future playback in the current frame of the contents to the neighboring node by the window size. As to performance evaluation, a comparative analysis was carried out with the aggressive caching scheme of unconditionally storing the received data, in the viewpoint of cache hit rate and transmission channel percentage. From the result of the simulation, it could have been confirmed that the proposed scheme has a high cache hit rate and a small number of transmission channels. Accordingly, Window-based Cooperative Caching Scheme can be an alternative scheme to increase the available energy and transmission efficiency of a node in MANETs.
\end{abstract}

Keywords: MANETs, Window, Cooperative Caching, Multimedia, Streaming

\section{Introduction}

Advancement of communication technology has enabled wide use of large high definition multimedia data. For this, large data is required to be transmitted along a reliable path for a long period of time. 5G mobile networks support a transmission speed of Giga bps or faster [1]. These networks require a communication infrastructure to be constructed, and much time and cost are required for the service to be provided. Also, it has a problem that service cannot be provided if no communication infrastructure is constructed or a failure occurs. An ad hoc network is a network comprised of communication terminals only [2][3]. As no communication infrastructure is required, the terminals should carry out all the works related to communication themselves. It is difficult to apply the scheme used for a wired network to Mobile ad-hoc networks (MANETs) that guarantee mobility of communication terminals due to their characteristics such as limited available energy, frequent change of transmission path resulting from node movement, and low transmission bandwidth. However, the demand of consumers for large, good quality multimedia contents is increasing. Multimedia contents encroach on the transmission bandwidth for the path between the server and the requesting node for a long period of time [4]. The more the service request rate increases, the more the required transmission bandwidth increases and the more the energy consumption of the nodes that comprise

\footnotetext{
${ }^{+}$Corresponding author. Tel.: +82-02-2164-4365; fax: +82-02-2164-4777

E-mail address: kpark@catholic.ac.kr
} 
the transmission path also increases. Such characteristics may cause a phenomenon of a delay or disconnection of the multimedia contents service.

Caching is a scheme that can resolve the availability problem of transmission bandwidth and node energy [5]. Caching scheme stores same contents not only in the server but also in multiple nodes. If a request for service is made, the relevant contents are provided by the node nearest to the requesting node among the nodes that have them in store. Accordingly, as the number of the transmission paths decreases when compared to the case where the contents are transmitted from the server, the network bandwidth and the energy consumption by node decrease. The storage capacity of a node is very small when compared with the total amount of the contents provided by the server. If a cache memory is fully filled, a new storage space should be secured by deleting the already stored contents. Accordingly, what contents should be stored and deleted is an important issue that determines caching performance. As nodes continuously move in MANETs, the transmission paths between nodes are variable in reference to time. The multimedia content that is large when compared to the content of another form requires much time for transmission. To reduce overlapping storage of contents, caching is carried out in general by designating a node. However, such a method may become an inefficient method as the distance between the caching node and the requesting node can become longer than the distance from the server to the requesting node due to node mobility. Also, if requests are concentrated on the caching node, a problem may arise where the use of transmission bandwidth and the load on node increase.

This paper proposes cooperative caching for multimedia contents in MANETs. Caching is carried out not by a designated specific node but by the nodes that comprise the transmission path. This feature can alleviate the problem where the transmission distance between the caching node and the requesting node becomes longer due to movement of node. As requests for contents service are not concentrated on a specific node, the load on node can be reduced. The process of finding a caching node is carried out only on the transmission path of the contents server without flooding request packets to the network. The requesting node makes a request to the nodes directly adjacent to it for additional frames of the multimedia contents and receives the same from it. This can reduce the impact of service delay or disconnection caused by transmission path and black hole problems.

This paper is organized as follows: Chapter 2 describes the related studies. In Chapter 3, the Windowbased caching scheme proposed in this paper is described. In Chapter 4, the performance of the proposed scheme is evaluated in the viewpoints of request rate, cache hit rate in reference to the window size and the number of transmission channels. Finally, Chapter 5 describes the conclusion.

\section{Related Work}

In MANETs, nodes are defined to have continuous mobility characteristic. The mobility model is diversely proposed making speed $\mathrm{v}$ and direction $\theta$ be dependent of time $\mathrm{t}$ [6][7]. When a node arrives at a defined destination, a new destination is selected. The model is divided into Random model and Temporal model depending on whether the newly selected speed and direction are related to the previous speed and direction or not. The Random model randomly selects a new destination and no correlation exists with the previous mobility. For this reason, the mobility characteristic is equally maintained until the node arrives at the destination, and the node speed or movement direction may rapidly change when it arrives at the destination. The Temporal model defines new mobility based on time differently from the Random model. The newly defined mobility is affected by the mobility until then, and changes may happen to the speed and direction at any time. A specific area may have a relatively high node density depending on the mobility model, and a black hole phenomenon where the connection with another node is cut may happen sometimes. This may affect the number of transmission channels and service durability.

MANETs require a process of continuously searching, setting and maintaining the path to guarantee optimum connection between nodes due to the mobility of node. This process is divided into proactive method and reactive method depending on whether the path is set or not when the service is requested [8][11]. The former is the method of periodically setting the transmission paths between the nodes existing in the whole network in advance irrespective of request for service. It can reduce time delay as no path searching process is required when service occurs. However, as it carries out a path setting process even 
when there is no request for service, network bandwidth and node energy are unnecessarily consumed. The Reactive method sets the path to the relevant node only when a request for service takes place. That is to say, as a path setting process is carried out only when required, it enables efficient use of transmission bandwidth and node energy. However, as a pass setting process is required before providing the service, service delay occurs. Though optimum efficiency is exhibited if transmission paths are managed in real time, as energy consumption increases, a problem of node availability arises. Though node availability and transmission bandwidth use efficiency increase if path management is carried out at a specified interval like a beacon, the latest path information may not be usable depending on the mobility.

Caching is a scheme of reducing service delay and the load on the server by providing the requested contents not from the server but from a node located close to the requesting node [12]-[16]. For a network comprised of a communication infrastructure and nodes, such a feature can be materialized by installing caching devices at the points of the communication infrastructure connected to the nodes. However, in the case of MANETs comprised of continuously moving nodes only, the boundary points between the communication infrastructure and the nodes cannot be distinguished. If caching is carried out by designating a specific node, as the distance between the caching node and the requesting node is variable, it is difficult to expect high efficiency. As the storage capacity or the processing capacity of a node is low when compared to that of the server, it is not suitable to accommodate multimedia contents. If a network is divided into a certain number of areas like clusters and a caching node is designated for each area, service delay can be reduced. In each area, the available time of the designated caching node rapidly decreases due to the service requests concentrated on it. Accordingly, a scheme is required that reduces load on node and increases availability by making multimedia contents to be stored being dispersed among nodes before being provided.

\section{Window-based Cooperative Caching Scheme}

This paper proposes a caching scheme that increases the availability of node energy by reducing the number of transmission paths for requested contents in MANETs. As MANETs are the networks comprised of nodes only, no separate communication equipment that takes charge of transmission exists. Each node sets links to other nodes located within its transmission range. All the nodes existing in a network carry out such a process and can set multi-hop transmission paths with other nodes by exchanging the link information with the adjacent nodes. The requested contents are sequentially stored in the nodes comprising the transmission path from the server and then delivered to the requesting node using the transmission method.

Let's call the number of contents provided from the server $\mathrm{N}$, the contents playback time PT(N), the contents server $\mathrm{S}$, the requesting node $\mathrm{R}$, the nodes comprising the transmission path $\mathrm{PN}$, and the number of transmission paths $\mathrm{H}$. Let's assume that the transmission path between the server $\mathrm{S}$ and the node $\mathrm{R}$ is set as < $\mathrm{S}-\mathrm{PN}(1)-\mathrm{PN}(2)-\ldots$ - PN(H-1) - R > at time t. The path node PN(i) forwards, to the child node PN(i+1), the contents $\mathrm{C}(\mathrm{i})$ transmitted from the parent node $\mathrm{PN}(\mathrm{i}-1)$ to send it to the descendant node $\mathrm{R}$. Therefore, the contents C(i) transmitted from the server are transmitted up to the node $\mathrm{R}$ repeatedly for $\mathrm{H}$ times. Though the path node $\mathrm{PN}(\mathrm{i})$ is not the requesting node, as it receives the transmitted contents $\mathrm{C}(\mathrm{i})$, it stores the relevant contents for the request to be made later. The transmission path at time $t$ and the transmission path at time $t+$ $\Delta t$ may differ from each other due to continuous movement of the nodes. Accordingly, the contents $\mathrm{C}(\mathrm{N})$ stored in the path node $\mathrm{PN}(\mathrm{i})$ is only a part of the contents $\mathrm{N}$ which correspond to the period comprising the transmission path, and $\mathrm{C}(\mathrm{N}) \leqq \mathrm{PT}(\mathrm{N})$. As this is not a method where caching is carried out only in designated nodes, it can reduce caching performance degradation and load on node caused by movement of node.

Fig. 1 shows an example of network topology at time t0. S represents the contents server and the solid lines show the links between each node. Let's assume that the node 6 requests for the contents $\mathrm{N}$ for the first time at time t0. Accordingly the contents $\mathrm{N}$ have not been transmitted before and no node has it in store. The requested packet is delivered to the server node via the node 5 which is a parent node comprising the path. The server $\mathrm{S}$ transmits the requested contents $\mathrm{N}$ to the node 5 which is a child node to send it to the node 6 . Though the node 5 is not the requesting node, as it is a node comprising the path, it stores the received contents and then transmits them to the child node 6 . In this process, the contents $\mathrm{N}$ are stored being overlapped in the requesting node and the nodes that comprise the transmission path. Let's assume that the 
network topology at time $t 0+\Delta t$ is as in Fig. 1 and the node 9 requests for the same contents $\mathrm{N}$ which the node 6 has requested at time t0. The request from the node 9 is received by the node 5 which is a parent node. As the node 5 has stored the contents $\mathrm{N}$ at time t0, it does not transmit this request packet to the server node $\mathrm{S}$ and transmits the contents in its store to the node 9 . In this case transmission of the contents $\mathrm{N}$ takes place not at $\{\mathrm{S}, 5,9\}$ but between $\{5,9\}$, the number of paths and bandwidth for transmission decrease.

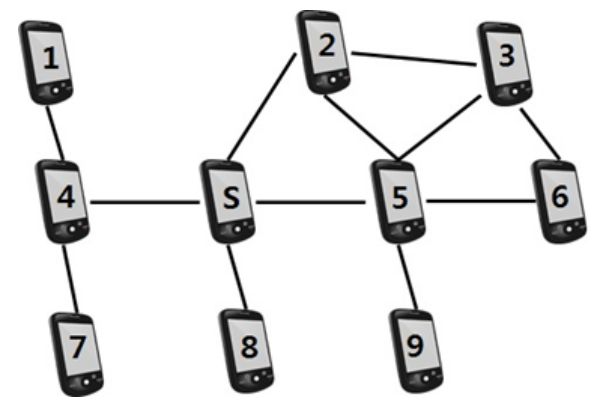

Fig. 1: An example of network topology at time t0

The contents transmitted in this paper are cached only in the path node and the requesting node. Let's say that the probability for the contents to be cached in the path node is $\mathrm{P}_{\mathrm{P}}$ and the probability for the contents to be cached in the requesting node is $\mathrm{P}_{\mathrm{R}}$. If the number of the transmission paths between the server and the requesting node is $\mathrm{H}$, the probability $\mathrm{P}_{\mathrm{C}}(\mathrm{N})$ for the transmitted contents $\mathrm{N}$ to be cached in a node is as in Equation (1).

$$
P_{C}(N)=\sum_{i=1}^{H-1} P_{P}+P_{R}
$$

Let's assume that the request of the node 6 for the contents $\mathrm{N}$ at time t0 is not the first request for the contents $\mathrm{N}$. In this case, the probability for the contents $\mathrm{N}$ to be in store in another node existing in the network increases. The contents $\mathrm{N}$ have a playback time of PT(N). In this paper, contents are assumed to be comprised of $\mathrm{M}$ frames and each node carries out caching for each frame. The probability for the m'th frame of the contents $\mathrm{N}$ to be cached in a node is as in Equation (2).

$$
P_{C}(N(m))=\sum_{i=1}^{H-1} P_{P}(N(m))+P_{R}(N(m))
$$

Mobility of node disconnects the path to the server or makes the distance to the caching node variable. This may cause delay or disconnection of real-time service of multimedia contents. To resolve this, the receiving node requests its adjacent nodes for additional frames of the contents frame currently played back and receives the same from them. The size of the frame additionally requested is defined as the window w. Accordingly, if the frame currently played back is the i'th frame F(i) of the contents, it additionally requests the adjacent nodes for a frame of size $\mathrm{F}(\mathrm{i}+\mathrm{w})$ and receives it. This process is carried out only for the adjacent nodes directed connected to the requesting nodes. Let's say that the number of adjacent nodes when the requesting node requests for the m'th frame of the contents $\mathrm{N}$ is Sm. When the Window scheme is used, the probability $\mathrm{P}_{\mathrm{L}}(\mathrm{N}(\mathrm{m}))$ for the requesting node to be already caching the contents $\mathrm{N}(\mathrm{m})$ is as in the following equation:

$$
P_{L}(N(m))=\sum^{S_{N(m)-w}} P_{C}(N(m))+\sum^{s_{N(m)-w+1}} P_{C}(N(m))+\cdots+\sum^{S_{N(m)-1}} P_{C}(N(m))=\bigcup_{i=N(m)-w}^{N(m)-1} \sum^{S_{i}} P_{C}(N(m))
$$

The caching capacity of a node is small when compared to that of the server. If a node can store $n$ contents, Equation (3) becomes like Equation (4). 


$$
P_{L}(N(m))=\frac{n \cdot P T(n)}{N \cdot P T(N)}\left(U_{i=N(m)-w}^{N(m)-1} \sum^{S i} P c(N(m))\right)
$$

The contents requested by a node can be provided by the requesting node, path node, or a node adjacent to the path node. If the cache hit probabilities in the requesting node, path node, and a node adjacent to the path node are Prequester, Ppath, and Psibling respectively, the probability for the contents to be provided not by the server but by a node, viz. the cache hit probability Pcache-hit-ratio is as Equation (5).

$$
P_{\text {cache-hit-ratio }}=P_{\text {requester }}+P_{\text {path }}+P_{\text {sibling }}=P_{L}(N(m))+\sum_{i=1}^{H-1} P_{L}(N(m))+U^{H}\left(\sum_{i=1}^{S_{N(m j}} P_{L}(N(m))\right)
$$

\section{Simulation and Analysis}

This section shows the result of the experiment that has analysed the performance of the proposed Window-based Cooperative Caching Scheme. It is assumed that only one contents server exists. The number of multimedia contents is 100, the playback time PT(n) is 30 seconds, and the playback rate per second SCBR is 1 Mbps. The cache size of a node is $\mathrm{nPT}(\mathrm{n})=300 \mathrm{SCBR}$. As to cache management algorithm, LRU (Least Recently Used) is used [17]. One hundred nodes with a transmission range of $150 \mathrm{~m}$ are located in a network of $800 \mathrm{~m} \mathrm{x} 800 \mathrm{~m}$ size. Content popularity is defined using Zipf's like distribution and the skew factor a is 0.85 [15]. The mobility of node is defined using Random waypoint (RWP); and the maximum speed is $8 \mathrm{~km} / \mathrm{h}$, average speed is $4 \mathrm{~km} / \mathrm{h}$ and the pause time is 5 seconds [6][7]. DSDV routing protocol is used and the routing performing interval is 1 second [8][10]. The time the simulation is carried out is 20000 seconds. In the initial state, nodes are located at random coordinates of the network.

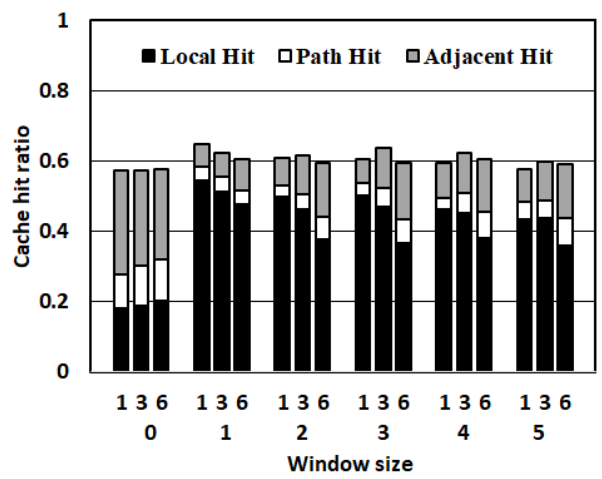

Fig. 2: Cache Hit Rate depending on Window Size and Request Rate in Aggressive Caching Scheme Environment

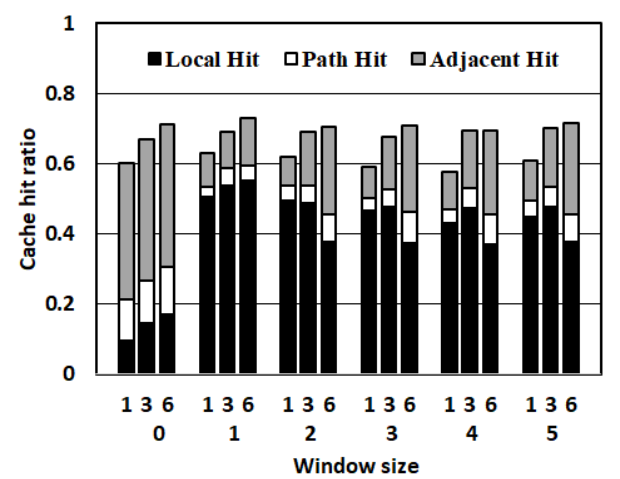

Fig. 3: Cache Hit Rate depending on Window Size and Request Rate in the proposed Caching Scheme Environment

Fig. 2 shows the cache hit rate in the aggressive caching scheme [18] which stores all contents received when the window size $\mathrm{w}=\{0,1,2,3,4,5\}$ and the request rate per minute $\lambda=\{1,3,6\}$. Local hit, path hit, and adjacent hit are the cache hit rates in the requesting node, path node and adjacent node respectively. The result of simulation shows that the correlation between the window size and the overall caching hit rate is low. However, it is shown that local hit greatly increases if Window scheme is used. The proposed scheme requests, in advance, the adjacent nodes for the frames, as big as the size of the window, preceding the frame of the contents currently played back to reduce the effect of a black hole phenomenon and transmission delay and store the same. As a result, local hit relatively increases to a great extent. Requests for contents based on popularity reduce the effect of cache miss resulting from the low cache capacity of node. If the request rate increases when the window size is the same, the local hit and overall cache hit rate decrease. It is because, the more the request rate increases, the more the renewal frequency of the frames stored in a cache increases.

Fig. 3 shows the cache hit rate depending on the window size and request rate in the proposed scheme where only the path node and the requesting node carry out caching. The proposed scheme exhibits excellent performance in the overall cache hit rate when compared with the aggressive caching. It can be seen from the results in Fig. 2 and Fig. 3 that, the bigger the window size is, the more the cache hit rate decreases. This is because, the more the request rate increases, the more the cache renewal frequency also increases. 
Accordingly, when seen from the viewpoint of the overall cache hit rate or local hit, it can be confirmed that, the smaller the window size is, the more excellent the performance is exhibited to be.

Fig. 4 shows the percentage of the transmission channel that decreases when a caching scheme is used. $\mathrm{P}(\lambda)$ shows the scheme where the transmitted contents are stored only in the path node and the requesting node, $\mathrm{A}(\lambda)$ the aggressive scheme where all contents are received irrespective of the location, and $\lambda$ means the request rate. If $\lambda$ is $1, \mathrm{P}(1)$ and $\mathrm{A}(1)$ have transmission channel percentages of about $27 \%$ and $53 \%$ respectively. If $\lambda$ is $3, \mathrm{P}(3)$ and $\mathrm{A}(3)$ have transmission channel percentages of about $43 \%$ and $50 \%$ respectively. If $\lambda$ is $6, \mathrm{P}(6)$ and $\mathrm{A}(6)$ have transmission channel percentages of about $30 \%$ and $46 \%$ respectively. It can be seen from the result of the simulation that window size has minor effect on the transmission channel percentage.

Fig. 5 shows the average number of transmission channels at every hour when $\lambda$ is 6 . NC( $\lambda)$ is the number of transmission channels when caching is not used. It can be seen from the simulation result that the numbers of transmission channels $\mathrm{P}(6)$ and $\mathrm{A}(6)$ require are about $30 \%$ and $38 \%$ respectively. The multimedia content which has a size of PT(n) should be played back from the first frame at all times. The LRU memorizes the latest information in cache. Therefore, if the cache renewal frequency increases, as the probability for the frame transmitted recently, not the first frame of the requested content, to be in store increases, cache miss probability increases. The Window Scheme plays the role of reducing the effect of such cache miss.

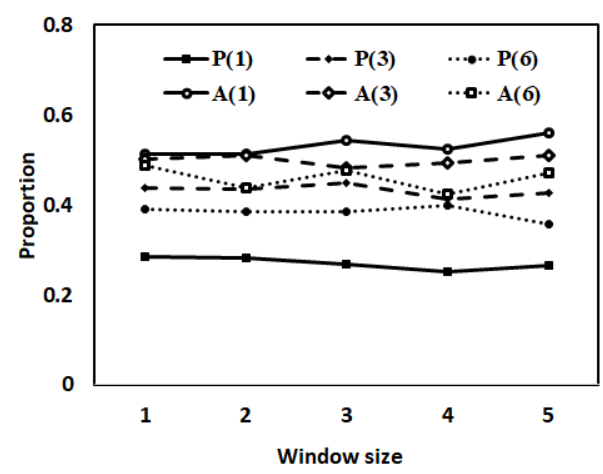

Fig. 4: Percentage of Number of Transmission Channels (Caching used / Caching not used)

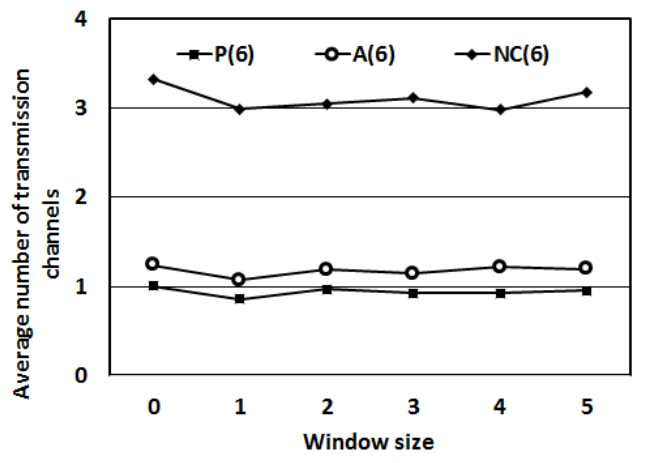

Fig. 5: Average Number of Transmission Channels

\section{Conclusion}

This paper proposes Cooperative Caching Scheme for Streaming Multimedia Contents in MANETs. The contents transmitted in the proposed scheme are cached and used in the path node and the requesting node. For the proposed caching scheme, the contents request rate, cache hit rate in the viewpoint of window size, and the number and percentage of transmission channels have been evaluated. The more the request rate increases, the more the cache renewal frequency increases. This degrades the cache hit rate and the use efficiency of transmission channel. To improve this, the requesting node requests the adjacent nodes for the contents frames, as big as the size of the set frame, required for subsequent playback and store the same. To evaluate the performance of the proposed scheme, a comparative analysis has been carried out with the aggressive caching scheme that unconditionally stores the data received. The result of the simulation indicates that the proposed Window Scheme shows a high cache hit rate and, in particular, great increase in the local cache hit rate. This can greatly reduce the number of channels required for transmission. Accordingly, the proposed caching scheme can be an alternative scheme that enables efficient use of node energy and transmission bandwidth.

\section{Acknowledgements}

This work was supported by the Catholic University of Korea, Research Grant, 2019. 


\section{References}

[1] M. Agiwal, A. Roy, A, and N. Saxena, "Next generation 5G wireless networks: A comprehensive survey,” IEEE Communications Surveys \& Tutorials, vol. 18, no. 3, pp. 1617-1655, 2016

[2] J. Wu and I. Stojenovic, “Ad hoc Networks,” IEEE Computer, pp. 29-31, 2004

[3] M. Conti and S. Giordano, "Mobile ad hoc networking: milestones, challenges, and new research directions," IEEE Communications Magazine, vol. 52, no. 4, pp. 85-96, 2014

[4] J. Yang, S. He, Y. Lin, and Z. Lv, "Multimedia cloud transmission and storage system based on internet of things," Multimedia Tools and Applications, vol. 76, no. 17, pp. 17735-17750, 2017

[5] P. Cao, and S. Irani, “Cost-aware www proxy caching algorithms,” In Usenix symposium on internet technologies and systems, vol. 12, no. 97, pp. 193-206, 1997

[6] T. Camp, J. Boleng, and V. Davies, “A Survey of Mobility Models for Ad Hoc Network Research,” Wireless Communications and Mobile Computing (WCMC): Special issue on Mobile Ad Hoc Networking Research, Trends and Applications, vol. 2, no. 5, pp. 483-502, 2002

[7] P. Nayak, and P. Sinha, "Analysis of random way point and random walk mobility model for reactive routing protocols for MANET using NetSim simulator,” In 2015 3rd International Conference on Artificial Intelligence, Modelling and Simulation (AIMS), IEEE, pp. 427-432, 2015

[8] Aggarwal, S. Gandhi, and N. Chaubey, "Performance analysis of AODV, DSDV and DSR in MANETS," International Journal of Distributed and Parallel Systems, vol. 2, no. 6, pp. 167-177, 2011

[9] H. Tran-Dang and D. Kim, "Energy-Efficient Cooperative Routing in Underwater Acoustic Sensor Networks," Journal of Advances in Information Technology, vol.10, no.4, pp.137-141, November 2019

[10] S. Mitra, R. Islam, K. Mukherjee, A. Das, and S. Nandi, “A Modified Algorithmic Approach of DSDV Routing Protocol for Wireless Ad Hoc Network,” IOSR Journal of Computer Engineering (IOSR-JCE), vol.14, no, 4, pp. 49-54, 2014

[11] N. Chaubey, A. Aggarwal, S. Gandhi, and K. A. Jani, "Performance analysis of TSDRP and AODV routing protocol under black hole attacks in manets by varying network size,” In Advanced Computing and Communication Technologies (ACCT), IEEE, pp. 320-324, 2015

[12] P. Srivastava and R. Kumar, "An efficient proxy adaptive gateway discovery algorithm based on quality of service parameters,” International Journal of Communication Networks and Distributed Systems, vol. 16, no. 3, pp. 261280, 2016

[13] G. Kioumourtzis, A. Gkamas, and C. Bouras, “Mobile Ad hoc networks (MANETs) for multimedia transmission,” In Encyclopedia of Information Science and Technology, pp. 6239-6248, 2015

[14] S. Lim, W. C. Lee, G. Cao, and C. R. Das, "Performance comparison of cache invalidation strategies for internetbased mobile ad hoc networks,” IEEE international conference on Mobile Ad-hoc and Sensor Systems (MASS), pp. 104-113, 2004

[15] L. Zhou, T, Zhang, X, Xu, Z. Zeng, and Y. Liu, "Broadcasting based neighborhood cooperative caching for content centric ad hoc networks,” In Communications in China (ICCC), 2015 IEEE/CIC International Conference on. IEEE, pp. 1-5, 2015

[16] R. A. Rehman and B. S. Kim, "LOMCF: Forwarding and Caching in Named Data Networking Based MANETs," IEEE Transactions on Vehicular Technology, vol. 66, no. 10, pp. 9350-9364, 2017

[17] N. Laoutaris, H. Che, and I. Stavrakakis, "The LCD interconnection of LRU caches and its analysis,” Performance Evaluation, vol. 63, no. 7, pp. 609-634, 2006

[18] B. Kim, I. Kim, and Y. Woo, "Hop-based exclusive neighborhood caching scheme for content centric IMANET," International Journal of Engineering \& Technology, 2018, vol. 7, pp. 143-146, 2018 